\title{
A Wideband IM3 Cancellation Technique using Negative Impedance for LNAs with Cascode Topology
}

\author{
Wei Cheng, Anne Johan Annema, Gerard J. M. Wienk and Bram Nauta \\ IC Design Group, CTIT Institute, University of Twente, Enschede, 7522NH, The Netherlands
}

\begin{abstract}
A negative impedance is used to enable distortion cancellation between the transconductor and the cascode transistor for LNAs with a cascode topology. As a proof of concept, a resistive feedback LNA using this IM3 cancellation technique in a standard $0.16 \mu \mathrm{m}$ CMOS process shows that for $0.1 \mathrm{GHz}$ to $1 \mathrm{GHz}$, improvements of $6.3 \mathrm{~dB}$ to $10 \mathrm{~dB}$ for IIP3 and $0.2 \mathrm{~dB}$ to $1 \mathrm{~dB}$ for gain are achieved without noise degradation. The power consumption for the LNA is increased by $2 \%$, and the die area by about $700 \mu \mathrm{m}^{2}$.

Index Terms - Low-noise amplifier (LNA), intermodulation distortion, output conductance nonlinearity, cascode transistor, IM3 cancellation, IIP3, CMOS.
\end{abstract}

\section{INTRODUCTION}

Much effort has been put in improving the linearity of LNAs. As a commonly used technique, an auxiliary path replicating the distortion of the main path is combined with the main LNA in a subtracting node. The auxiliary path can be transistors biased in weak inversion [1-2], transistors biased in saturation region as a nonlinear resistor [3] or current sources that injects IM2 to suppress IM3 [4]. The limitations of most reported linearization techniques mainly are due to:

1) only focusing on transconductance nonlinearity, neglecting nonlinearity related to output conductance [5].

2) neglecting the distortion generated by the cascode transistor.

Neglecting output conductance related distortion and neglecting the distortion contribution of cascode transistors is valid for older technologies with longchannel devices and high supply voltage. However, in deep submicron CMOS technologies, typically the output resistance of the transconductor stage is relatively low: the IM3 distortion contribution from the cascode transistor then may become dominant. On top of that, the low supply voltage together with high gain operation tends to push the cascode transistor out of the deep saturation region. This results in a very significant increase of the third-order output conductance nonlinearity term and the crossmodulation nonlinearities, which causes the increase of distortion generated by the cascode transistor [7].
In this paper we present a wideband IM3 cancellation technique that takes into account the distortion of the cascode transistor and all the third-order nonlinearity contributions related to the transconductance and the output conductance. A negative impedance is used to enable cancellation between the distortion current of the transconductor and the cascode transistor. Without loss of generality, we apply this IM3 cancellation technique to a resistive feedback wideband LNA. Section II presents an analysis to explain the proposed IM3 cancellation and presents a short discussion on the effect on gain and noise. Section III shows both simulation and measurement results to verify the theory.

\section{THEORY OF IM3 CANCELLATION USING NEGATIVE IMPEDANCE}

In the resistive feedback LNA shown in Fig. $1, M_{1 a} / M_{1 b}$ is the transconductor stage while the cascode transistor $\mathrm{M}_{2 \mathrm{a}} / \mathrm{M}_{2 \mathrm{~b}}$ increases the output impedance and improves the isolation between input and output. The shunt feedback resistor $R_{f}$ is used to match to the source resistance $R_{s}$.

To improve the linearity we apply a negative impedance $\left(Y_{\text {neg }}=G_{\text {neg }}+j \omega C_{\text {neg }}\right.$ ) between the drain of $\mathrm{M}_{1 \mathrm{a}} / \mathrm{M}_{1 \mathrm{~b}}$ as shown in Fig. 2. The IM3 distortion current of transistor $M_{x}$ is due to the voltage swings at the transistor terminals, where we neglect the effect of a source-bulk signal for simplicity reasons only. This results in a $3^{\text {rd }}$ order current $i_{d}^{M x}=g_{m 3} v_{g s}^{3}+g_{x 21} v_{g s}^{2} v_{d s}+g_{x 12} v_{g s} v_{d s}^{2}+g_{d 3} v_{d s}^{3}$, for

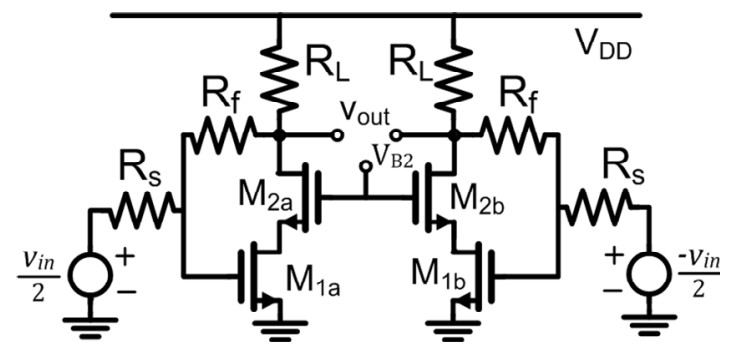

Fig. 1. Schematic of a conventional differential resistive feedback LNA. 


$$
\begin{aligned}
& v_{\text {out }}^{I M 3}=-\frac{2\left(R_{f}+R_{s}\right) R_{L}}{D_{v}}\left[g_{m}^{M_{2}} i_{d}^{M_{1}}+\left(Y_{d s}^{M_{1}}+2 Y_{\text {neg }}\right) i_{d}^{M_{2}}\right] \\
& \text { where } D_{v}=g_{m}^{M_{2}}\left(R_{f}+R_{L}+R_{s}+g_{m}^{M_{1}} R_{L} R_{S}\right)+\left(R_{f}+R_{L}+R_{S}\right)\left(Y_{d s}^{M_{1}}+2 Y_{\text {neg }}\right) \\
& i_{d}^{M_{1}}=f_{d}^{M_{1}}\left(v_{g s}^{M_{1 a}}, v_{d s}^{M_{1 a}}\right)=f_{d}^{M_{1}}\left[\frac{\left(R_{f}+R_{L}\right)\left(g_{m}^{M_{2}}+Y_{d s}^{M_{1}}+2 Y_{n e g}\right) v_{i n}}{2 D_{v}}, \frac{-g_{m}^{M_{1}}\left(R_{f}+R_{L}\right) v_{i n}}{2 D_{v}}\right] \\
& i_{d}^{M_{2}}=f_{d}^{M_{2}}\left(v_{g s}^{M_{2 a}}, v_{d s}^{M_{2 a}}\right)=f_{d}^{M_{2}}\left[\frac{g_{m}^{M_{1}}\left(R_{f}+R_{L}\right) v_{i n}}{2 D_{v}}, \frac{g_{m}^{M_{1}}\left(R_{f}+R_{L}-g_{m}^{M_{2}} R_{f} R_{L}\right)+R_{L}\left(g_{m}^{M_{2}}+Y_{d s}^{M_{1}}+2 Y_{n e g}\right)}{2 D_{v}}\right] \\
& G_{n e g} \in\left[\frac{-g_{d s}^{M_{1}}-g_{m}^{M_{2}}}{2}, \frac{-g_{d s}^{M_{1}}}{2}\right], C_{n e g} \approx-0.5 C_{d s}^{M_{1}} \\
& A_{V}=\frac{R_{L}\left[g_{m}^{M_{2}}\left(1-g_{m}^{M_{1}} R_{f}\right)+Y_{d s}^{M_{1}}+2 Y_{n e g}\right]}{D_{v}} \approx \frac{R_{L}\left(-g_{m}^{M_{1}} g_{m}^{M_{2}} R_{f}+Y_{d s}^{M_{1}}+2 Y_{n e g}\right)}{D_{v}} \\
& N F=1+\frac{\left[g_{m}^{M_{2}} R_{L}\left(R_{f}+R_{s}\right)\right]^{2} \gamma g_{m}^{M_{1}}}{R_{s} D_{n}^{2}}+\frac{\left[R_{L}\left(g_{m}^{M_{2}}+g_{m}^{M_{1}} g_{m}^{M_{2}} R_{s}+Y_{d s}^{M_{1}}+2 Y_{n e g}\right)\right]^{2} R_{f}}{R_{s} D_{n}^{2}}+\frac{\left[\left(R_{f}+R_{s}\right)\left(g_{m}^{M_{2}}+Y_{d s}^{M_{1}}+2 Y_{n e g}\right)\right]^{2} R_{L}}{R_{s} D_{n}^{2}} \\
& +\frac{\left[R_{L}\left(R_{f}+R_{s}\right)\left(Y_{d s}^{M_{1}}+2 Y_{n e g}\right)\right]^{2} \gamma g_{m}^{M_{2}}}{R_{s} D_{n}^{2}}+\frac{\left[g_{m}^{M_{2}} R_{L}\left(R_{f}+R_{s}\right)\right]^{2}\left|G_{n e g}\right|}{R_{s} D_{n}^{2}} \\
& \text { where } D_{n}=g_{m}^{M_{2}}\left(1-g_{m}^{M_{1}} R_{f}\right)+Y_{d s}^{M_{1}}+2 Y_{\text {neg }} \approx-g_{m}^{M_{1}} g_{m}^{M_{2}} R_{f}+Y_{d s}^{M_{1}}+2 Y_{\text {neg }}
\end{aligned}
$$

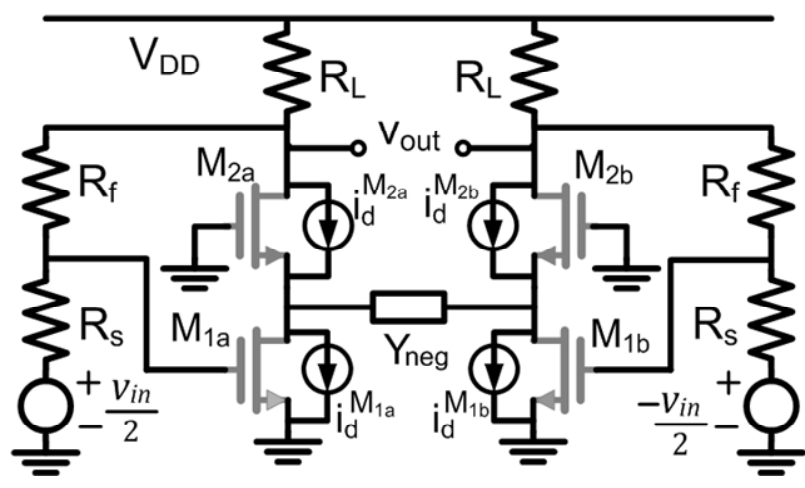

Fig. 2. Circuit model for the distortion and gain analysis .

which a short hand notation $i_{d}^{M_{x}}=f_{d}^{M_{x}}\left(v_{g s}^{M_{x}}, v_{d s}^{M_{x}}\right)$ will be used in the remainder of this paper. The exact function that describes the transistor nonlinearity is not relevant in this paper. Given the differential circuit topology we assume $i_{d}^{M_{1 a}}=-i_{d}^{M_{1 b}}=i_{d}^{M_{1}}$ and $i_{d}^{M_{2 a}}=-i_{d}^{M_{2 b}}=i_{d}^{M_{2}}$.

To determine the LNA's IM3, a symbolic harmonic balance like analysis with truncated terms was carried out, yielding the closed form solution for the IM3 shown in (1). In (1) the $3^{\text {rd }}$ order distortion currents $i_{d}^{M_{1}}$ and $i_{d}^{M_{2}}$ are according to the previously introduced $i_{d}^{M_{x}}=f_{d}^{M_{x}}\left(v_{g s}^{M_{x}}, v_{d s}^{M_{x}}\right)$, which in this circuit expands to (2-3). It now follows from (2-3) that for $Y_{n e g}>-0.5\left(g_{m}^{M_{2}}+Y_{d s}^{M_{1}}\right)$ the polarity of the voltage swings for $\mathrm{M}_{1 \mathrm{a}} / \mathrm{M}_{1 \mathrm{~b}}$ and $\mathrm{M}_{2 \mathrm{a}} / \mathrm{M}_{2 \mathrm{~b}}$ are unchanged, and hence so is the phase of the IM3 currents of the transistors. At the same time, (1) shows that for $Y_{\text {neg }}<$ $-0.5 Y_{d s}^{M_{1}}$ the distortion components of the transistors are subtracted. Combining these results yields a region defined by (4) in which IM3 contributions of $M_{1}$ and $M_{2}$ (partially or fully) cancel. Note that doing so, the distortion of the transconductor transistors is canceled using the distortion contribution of the cascode transistors.
The small signal voltage gain can be derived from the model in Fig. 2, resulting in (5). Since $Y_{\text {neg }}$ increases the overall output impedance of the transconductor $\mathrm{M}_{1 \mathrm{a}} / \mathrm{M}_{1 \mathrm{~b}}$, a higher gain is resulted. The noise figure (NF) is calculated by the model shown in Fig. 3, where we include the thermal noise current of $\mathrm{M}_{1 \mathrm{a}} / \mathrm{M}_{1 \mathrm{~b}}, \mathrm{M}_{2 \mathrm{a}} / \mathrm{M}_{2 \mathrm{~b}}$ and $\mathrm{Y}_{\text {neg }}$ $\left(i_{\text {th }}^{Y_{\text {neg }}}=4 k T\left|G_{\text {neg }}\right|\right)$ and the thermal noise voltage of $\mathrm{R}_{\mathrm{f}}, \mathrm{R}_{\mathrm{s}}$ and $R_{L}$. The calculated NF is given by (6), where the five terms account for the thermal noise from respectively $M_{1 a} / M_{1 b}, R_{f}, R_{L}, M_{2 a} / M_{2 b}$ and $Y_{\text {neg. Compared to the }}$ situation without an $\mathrm{Y}_{\text {neg, }}$, when the IM3 cancellation condition (4) is met, the denominator $D_{n}^{2}$ is increased and the nominator of the second term and the third term are decreased. This results in decreases of NF contribution from $M_{1 a} / M_{1 b}, R_{f}$ and $R_{L}$. Since $Y_{\text {neg }}$ increases the overall output impedance of the transconductor $\mathrm{M}_{1 \mathrm{a}} / \mathrm{M}_{1 \mathrm{~b}}$, less noise contribution can come from the cascode transistor $\mathrm{M}_{2 \mathrm{a}} / \mathrm{M}_{2 \mathrm{~b}}$. Therefore, it can be concluded that although a $\mathrm{Y}_{\text {neg }}$ circuit introduces extra noise (last term in (6)), it also reduces the NF contribution from $\mathrm{M}_{1 \mathrm{a}} / \mathrm{M}_{1 \mathrm{~b}}, \mathrm{M}_{2 \mathrm{a}} / \mathrm{M}_{2 \mathrm{~b}}, \mathrm{R}_{\mathrm{f}}$ and $R_{L}$. As a result, the effect on NF by a $Y_{\text {neg }}$ circuit can be small.

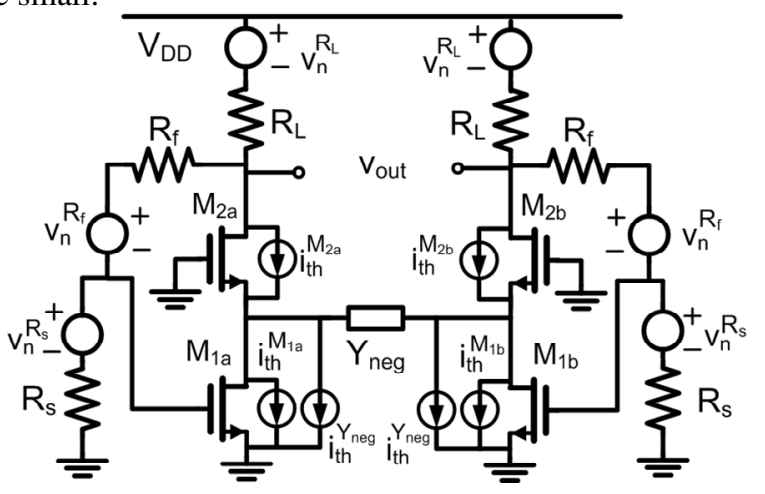

Fig. 3. Circuit model for calculating noise. 


\section{LNA DESIGN AND EXPERIMENTAL RESULTS}

To prove this IM3 cancellation concept, the LNA in Fig. 4 is implemented in a standard $0.16 \mu \mathrm{m}$ CMOS process. The negative impedance is implemented by the crosscoupled pair $\mathrm{M}_{4 \mathrm{a}} / \mathrm{M}_{4 \mathrm{~b}}$ with source degeneration provided by capacitor $\mathrm{C}_{\mathrm{s}}(1.6 \mathrm{pF})$ and current source $\mathrm{M}_{5 \mathrm{a}} / \mathrm{M}_{5 \mathrm{~b}}$ [8]. The capacitor $\mathrm{C}_{\mathrm{f}}(1.57 \mathrm{pF})$ and $\mathrm{R}_{\mathrm{f}}(370 \Omega)$ provide the shunt feedback. The $\mathrm{Y}_{\text {neg }}$ circuit provides an almost-constant negative resistance and a frequency dependent negative capacitance (decreasing with frequency).

We designed the $Y_{\text {neg }}$ circuit for full IM3 cancellation at $1 \mathrm{GHz}$. A buffer and a resistive attenuator are put in parallel on-chip after the LNA for noise/gain and IIP3 measurement. The IIP3 is extrapolated from $-30 \mathrm{dBm}$ to $-20 \mathrm{dBm}$. The chip microphotograph is shown in Fig. $4 \mathrm{~b}$. The LNA occupies $0.00295 \mathrm{~mm}^{2}$ active area, of which $25 \%$ is taken by the $\mathrm{Y}_{\text {neg }}$ circuit. Packaged chips were measured on PCB boards. Two off-chip baluns were used at the input and output of the chip for single-ended-todifferential conversion. By switching on/off of the $Y_{\text {neg }}$ circuit, we measure the effect of $\mathrm{Y}_{\text {neg }}$ on LNA performance.

The measured and simulated $\mathrm{S}_{11}, \mathrm{NF}$ and voltage gain is shown in Fig. 5. For $0.1 \mathrm{GHz}$ to $1 \mathrm{GHz}$, the $Y_{\text {neg }}$ circuit introduces no degradation on NF while it improves $S_{11}$ by $1-3 \mathrm{~dB}$ and improves gain by $0.2-1 \mathrm{~dB}$. Below $0.3 \mathrm{GHz} \mathrm{S}_{11}$ becomes $>-10 \mathrm{~dB}$ because the impedance of $\mathrm{C}_{\mathrm{f}}$ starts to block the shunt feedback. This can be improved by using a larger $\mathrm{C}_{\mathrm{f}}$. Note that this LNA is not optimized for very low $\mathrm{NF}$ as we only focus on demonstrating the IM3 cancellation technique.

To verify the robustness against process spread, the bias current of $\mathrm{Y}_{\text {neg }}\left(\mathrm{I}_{\mathrm{Yneg}}\right)$ is swept within $\pm 100 \%$ variation of the optimal value. Fig. 6a shows the IIP3 improvement with respect to the circuit without $Y_{\text {neg }}$ as a function of $\mathrm{I}_{\text {Yneg }}$ normalized to the optimum $\mathrm{I}_{\text {Yneg,opt }}$; this

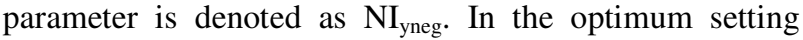
hence $\mathrm{NI}_{\mathrm{yneg}}=100 \%$. Fig. $6 \mathrm{a}$ shows that for wide bias variation $\left(\mathrm{NI}_{\text {yneg }}=-30 \%\right.$ to $\left.\mathrm{NI}_{\mathrm{yneg}}=+100 \%\right),+6 \mathrm{~dB}$ IIP3 improvement is achieved at $1 \mathrm{GHz}$. The power overhead of this technique is depicted in Fig. 6 b.
Fig. 7a shows the frequency dependence of the IM3 cancellation technique, for $\mathrm{NI}_{\text {yneg }}=100 \%$, on both IIP3 and on $\mathrm{P}_{1 \mathrm{~dB}}$. The measurements and simulation results in Fig. 7 show a weak frequency dependence in the IIP3 improvement and hence quite good robustness. Fig. 8 shows the measured HD1 and IM3 output at the optimal

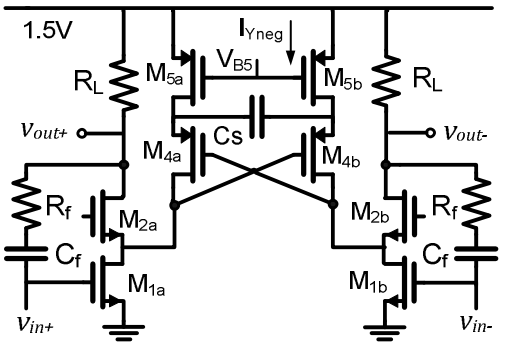

(a)

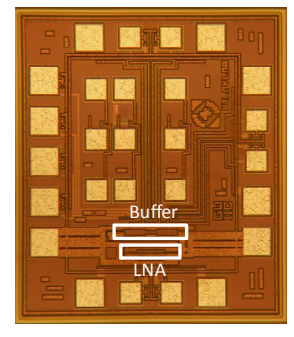

(b)
Fig. 4. LNA using $Y_{\text {neg }}$ for IM3 cancellation. (a) Schematic. (b) Microphotograph of the fabricated chip.
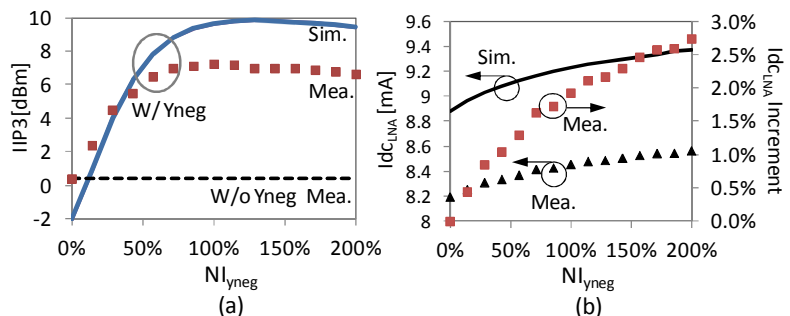

Fig. 6. Measured and simulated (a) IIP3 at $1 \mathrm{GHz}$ and (b) DC current consumption of LNA as a function of the nominated bias current of $Y_{\text {neg. }}$.

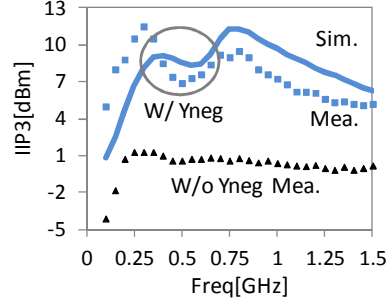

(a)

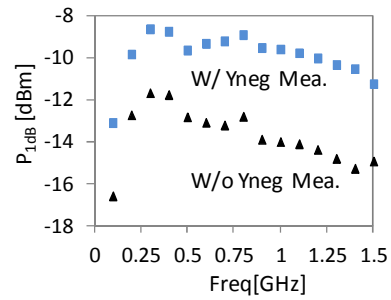

(b)
Fig. 7. Measured (a) IIP3 and (b) input $\mathrm{P}_{1 \mathrm{~dB}}$ as a function of RF frequency

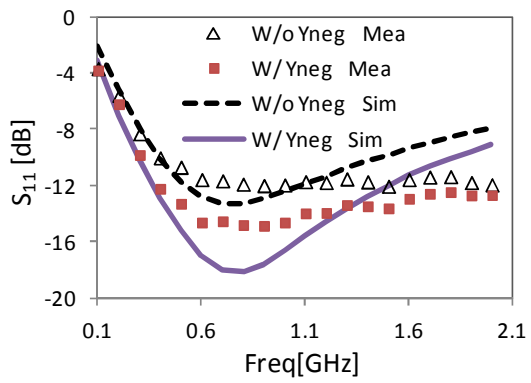

(a)

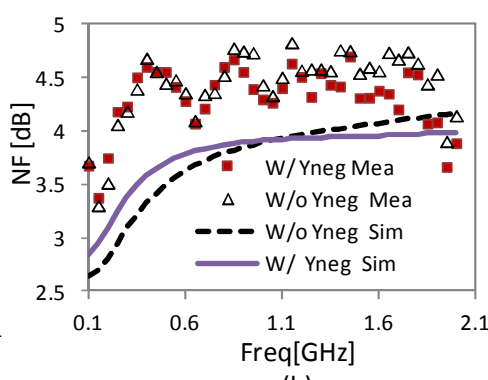

(b)

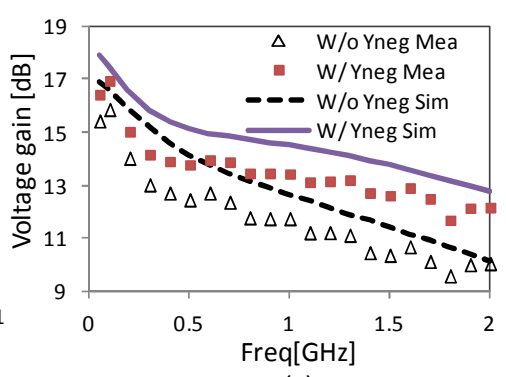

(c)

Fig. 5. Measured and simulated (a) $\mathrm{S}_{11}$, (b) NF and (c) Voltage gain. 


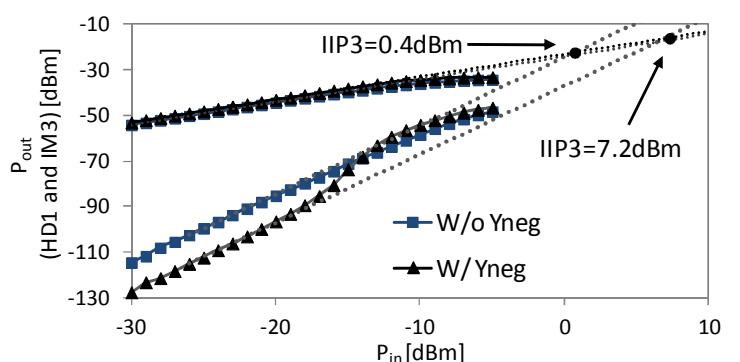

Fig. 8. Measured HD1 and IM3 at $1 \mathrm{GHz}$ as a function of input power.

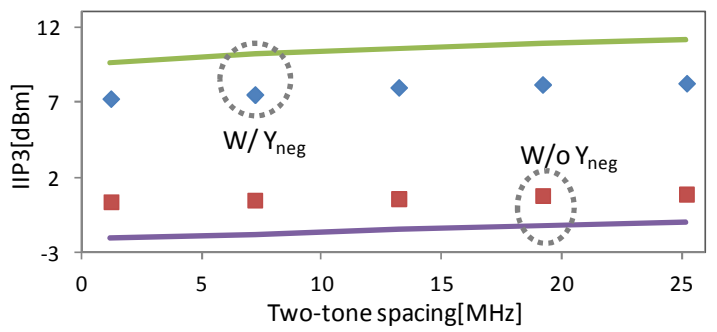

Fig. 9. Measured IIP3 at $1 \mathrm{GHz}$ as a function of two-tone spacing. Line for simulations and symbol for measurements.

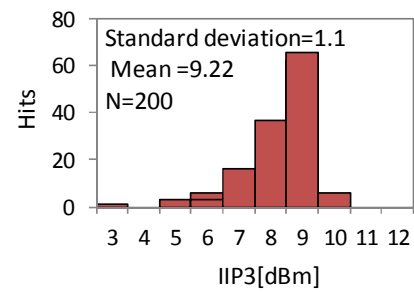

(a)

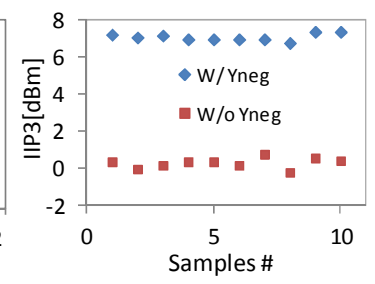

(b)
Fig. 10. Effect of mismatches and process spread on IIP3 at 1GHz. (a) 200-time Monte Carlo simulation results of IIP3 with $\mathrm{Y}_{\text {neg }}\left(\mathrm{NI}_{\text {yneg }}=100 \%\right)$ and (b) measured IIP3 of ten dies for LNA with and without $Y_{\text {neg. }}$.

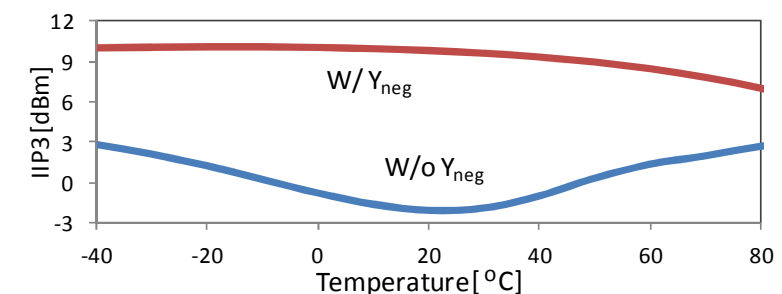

Fig. 11. Simulated IIP3 as a function of temperature for LNA with and without $Y_{\text {neg. }}$.

bias value of $\mathrm{Y}_{\text {neg }}\left(\mathrm{NI}_{\mathrm{Yneg}}=100 \%\right)$. The IM3 curve starts to show $5^{\text {th }}$ order behavior for Pin>-18dBm due to the transistors' higher-order nonlinearities that kick in at high input magnitudes. Fig. 9 presents IIP3 simulations results and IIP3 measurements as a function of the two-tone spacing, showing that the IM3 cancellation technique is not sensitive to two-tone spacing.

To estimate the overall effect of process spread and mismatch on this IM3 cancellation, a 200-time MonteCarlo simulation is performed for an RF signal at $1 \mathrm{GHz}$.
Fig. 10a shows that the mean IIP3 is $9.2 \mathrm{dBm}$ at the optimal bias value of $\mathrm{Y}_{\text {neg }}\left(\mathrm{NI}_{\mathrm{Yneg}}=100 \%\right)$ (the nominal value is $9.6 \mathrm{dBm}$ ), which is $9 \mathrm{~dB}$ higher than the LNA without $\mathrm{Y}_{\text {neg. }}$. This shows good robustness of this IM3 cancellation technique. The measurement results of ten LNA samples shows $+6.2 \mathrm{~dB}$ IIP3 improvement at $1 \mathrm{GHz}$ as shown in Fig.10b. The simulation results in Fig. 11 show that the LNA with the $Y_{\text {neg }}$ circuit provides a constant IIP3 from $-40^{\circ} \mathrm{C}$ to $40^{\circ} \mathrm{C}$ and starts to decreases as the temperature higher than $40^{\circ} \mathrm{C}$.

\section{CONCLUSION}

This paper presents a wideband IM3 cancellation technique using a negative impedance, applied to a wide band cascode LNA. Using a suitable negative impedance, the distortion current generated by the cascode transistor cancels the distortion from the transconductor. The negative impedance also increases gain while its effect on NF can be minimal. For a resistive feedback LNA fabricated in a standard $0.16 \mu \mathrm{m}$ CMOS process, for $0.1 \mathrm{GHz}$ to $1 \mathrm{GHz}$ this IM3 cancellation technique improves IIP3 by $6.3 \mathrm{~dB}$ to $10 \mathrm{~dB}$, gain by 0.2 to $1 \mathrm{~dB}$ and $\mathrm{P}_{1 \mathrm{~dB}}$ by $+3 \mathrm{~dB}$ while NF is not degraded, at a low area and power penalty. Robustness of this cancellation technique is demonstrated both in simulation and in measurements.

\section{ACKNOWLEDGEMENT}

The authors thank NXP for chip fabrication, and G. van der Weide and H. de Vries for their help.

\section{REFERENCES}

[1] T. W. Kim, "A common-gate amplifier with transconductance nonlinearity cancellation and its highfrequency analysis using the Volterra series," IEEE Trans. Microw. Theory Tech., vol.57, no. 6, pp.1461-1469, 2009.

[2] T.-S. Kim, et al., "Linearization of differential CMOS low noise amplifier using cross-coupled post distortion canceller" IEEE RFIC, pp.83-86, 2008.

[3] H. Zhang, et al., "A low-power linearized ultra-wideband LNA design technique," IEEE JSSC, no. 2, pp.320-330, 2009.

[4] S. Lou, et al, "A linearization technique for RF receiver front-end using second-order-intermodulation injection" IEEE JSSC, vol. 43, no. 11, pp.2404-2412, Nov. 2008.

[6] H. Zhang, et al, "Linearization techniques for CMOS low noise amplifiers: A Tutorial," IEEE Trans. Circuits and Systems I, vol. 58, no. 1, pp.22-36, Jan. 2011.

[7] W. Cheng, et al, "Noise and nonlinearity modeling of active mixers for fast and accurate estimation" IEEE Trans. Circuits and Systems I, no. 2, pp.276-289, Feb. 2011.

[8] C. Tilhac, et al, "A Tunable bandpass BAE-filter architecture using negative capacitance circuitry," IEEE RFIC, pp.605-608, 2008. 